\title{
New Oscillation Results of Second-Order Damped Dynamic Equations with $p$-Laplacian on Time Scales
}

\author{
Yang-Cong Qiu' ${ }^{1}$ and Qi-Ru Wang ${ }^{2}$ \\ ${ }^{1}$ School of Humanities \& Social Science, Shunde Polytechnic, Foshan, Guangdong 528333, China \\ ${ }^{2}$ School of Mathematics \& Computational Science, Sun Yat-Sen University, Guangzhou, Guangdong 510275, China \\ Correspondence should be addressed to Qi-Ru Wang; mcswqr@mail.sysu.edu.cn
}

Received 16 May 2014; Accepted 21 October 2014

Academic Editor: Wei Nian Li

Copyright ( $) 2015$ Y.-C. Qiu and Q.-R. Wang. This is an open access article distributed under the Creative Commons Attribution License, which permits unrestricted use, distribution, and reproduction in any medium, provided the original work is properly cited.

By employing a generalized Riccati technique and functions in some function classes for integral averaging, we derive new oscillation criteria of second-order damped dynamic equation with $p$-Laplacian on time scales of the form $\left(r(t) \varphi_{\gamma}\left(x^{\Delta}(t)\right)\right)^{\Delta}+$ $p(t) \varphi_{\gamma}\left(x^{\Delta}(t)\right)+f(t, x(g(t)))=0$, where the coefficient function $p(t)$ may change sign. Two examples are given to demonstrate the obtained results.

\section{Introduction}

In 1988, the theory of time scales was introduced by Hilger in his Ph.D. thesis [1] in order to unify continuous and discrete analysis; see also [2]. In recent years, there has been much research activity concerning the oscillation of solutions of dynamic equations on time scales; for example, see [3-19] and the references therein.

Došlý and Hilger [10] considered the second-order dynamic equation

$$
\left(p(t) x^{\Delta}(t)\right)^{\Delta}+q(t) x(\sigma(t))=0
$$

and they gave some necessary and sufficient conditions for the oscillation of all solutions as (1) on unbounded time scales. Erbe et al. [11] studied the second-order nonlinear dynamic equation

$$
\left(p(t) x^{\Delta}(t)\right)^{\Delta}+q(t)\left(f \circ x^{\sigma}\right)=0 .
$$

By means of generalized exponential functions and generalized Riccati transformation techniques, some oscillation criteria were given for (2). Del Medico and Kong [8,9] employed the Riccati transformation and gave sufficient conditions for Kamenev-type oscillation criteria of (1) on a measure chain. Wang [18] considered the second-order nonlinear damped differential equation

$$
\begin{aligned}
& \left(a(t) \psi(x(t)) k\left(x^{\prime}(t)\right)\right)^{\prime}+p(t) k\left(x^{\prime}(t)\right) \\
& \quad+q(t) f(x(t))=0, \quad t \geq t_{0},
\end{aligned}
$$

used the generalized Riccati transformations, and established new oscillation criteria of (3). Saker et al. [16] studied the second-order damped dynamic equation with damping

$$
\left(a(t) x^{\Delta}(t)\right)^{\Delta}+p(t) x^{\Delta^{\sigma}}(t)+q(t)\left(f \circ x^{\sigma}\right)=0,
$$

and they gave some oscillation criteria. Agarwal et al. [5] studied the second-order half-linear dynamic equation

$$
\left(r(t)\left(x^{\Delta}(t)\right)^{\gamma}\right)^{\Delta}+p(t) x^{\gamma}(t)=0, \quad t \in[a, b],
$$

and established Philos-type oscillation criteria. Huang and Wang [13] considered the second-order nonlinear dynamic equation

$$
\left(p(t) x^{\Delta}(t)\right)^{\Delta}+f(t, x(\sigma(t)))=0 .
$$


The authors extended the results in $[8,9]$, and established some new Kamenev-type oscillation criteria. Qiu and Wang [14] studied the dynamic equations of a more general form

$$
\left(p(t) \psi(x(t)) k \circ x^{\Delta}(t)\right)^{\Delta}+f(t, x(\sigma(t)))=0
$$

and established some similar oscillation criteria.

Şenel [17] discussed the oscillation of second-order damped dynamic equations of the following form:

$$
\left(r(t)\left(x^{\Delta}(t)\right)^{\gamma}\right)^{\Delta}+p(t)\left(x^{\Delta}(t)\right)^{\gamma}+f(t, x(g(t)))=0
$$

on a time scale $\mathbb{T}$ satisfying inf $\mathbb{T}=t_{0}$ and sup $\mathbb{T}=\infty$, where $p \in C_{r d}(\mathbb{T},[0, \infty))$. However, it seemed that several mistakes had been made and the obtained theorems and corollaries were incorrect. Qiu and Wang [15] corrected some mistakes in [17] and established some Kamenev-type oscillation criteria of (8) by employing functions in some function classes and a similar generalized Riccati transformation as used in $[13,18,19]$.

Hassan and Kong [12] considered the second-order dynamic equation with $p$-Laplacian and damping

$$
\left(r(t) \varphi_{\gamma}\left(x^{\Delta}(t)\right)\right)^{\Delta}+p(t) \varphi_{\gamma}\left(x^{\Delta \sigma}(t)\right)+q(t) f\left(x^{\sigma}(t)\right)=0,
$$

where $\varphi_{\gamma}(u):=|u|^{\gamma-1} u, \gamma>0$. The authors used the notations

$$
R(t)=r(t) a(t), \quad Q(t)=q(t) a(t),
$$

where $a(t):=e_{p(t) / r^{\sigma}(t)}\left(t, t_{0}\right)$, by which (9) can be written into the form

$$
\left(R(t) \varphi_{\gamma}\left(x^{\Delta}(t)\right)\right)^{\Delta}+Q(t) f\left(x^{\sigma}(t)\right)=0 \text { for } t \in\left[t_{0}, \infty\right)_{\mathbb{T}},
$$

and established some oscillation criteria of (9).

In this paper, we will consider the second-order damped dynamic equation with $p$-Laplacian

$$
\left(r(t) \varphi_{\gamma}\left(x^{\Delta}(t)\right)\right)^{\Delta}+p(t) \varphi_{\gamma}\left(x^{\Delta}(t)\right)+f(t, x(g(t)))=0
$$

on a time scale $\mathbb{T}$ satisfying inf $\mathbb{T}=t_{0}$ and sup $\mathbb{T}=\infty$, where the coefficient function $p(t)$ may change sign. Throughout this paper, we will assume that

(C1) $r \in C_{r d}(\mathbb{T},(0, \infty)), \varphi_{\gamma}(u):=|u|^{\gamma-1} u, \gamma>0$;

(C2) $p \in C_{r d}(\mathbb{T}, \mathbb{R}),-p(t) / r(t)$ is positively regressive, which means $1-\mu(t) p(t) / r(t)>0$, and

$$
\int_{t_{0}}^{\infty}\left(\frac{e_{-p / r}\left(t, t_{0}\right)}{r(t)}\right)^{1 / \gamma} \Delta t=\infty
$$

(C3) $g \in C(\mathbb{T}, \mathbb{T})$, and for any $t \in \mathbb{T}$,

$$
g(t) \geq \begin{cases}\sigma(t), & 0<\gamma<1 \\ t, & \gamma \geq 1\end{cases}
$$

(C4) $f \in C(\mathbb{T} \times \mathbb{R}, \mathbb{R})$, and there exists a function $q \in$ $C_{r d}(\mathbb{T},[0, \infty))$ such that $u f(t, u) \geq q(t) u^{\gamma+1}$.

The rest of this paper is organized as follows. In Section 2, we give some basic lemmas. In Section 3, we derive new oscillation criteria for (12). In Section 4, two examples are included to show the significance of the results.

\section{Basic Lemmas}

Preliminaries about time scale calculus can be found in $[3,4,6,7]$ and hence we omit them here. Note that we have the following properties for some typical time scales, respectively:

(1) $\mathbb{T}=[0, \infty)$,

$$
\begin{gathered}
\sigma(t)=\rho(t)=t, \quad f^{\Delta}(t)=f^{\prime}(t), \\
\int_{a}^{b} f(t) \Delta t=\int_{a}^{b} f(t) d t
\end{gathered}
$$

(2) $\mathbb{T}=\mathbb{N}_{0}$,

$$
\begin{gathered}
\sigma(t)=t+1, \quad \rho(t)=t-1, \\
f^{\Delta}(t)=f(t+1)-f(t), \\
\int_{a}^{b} f(t) \Delta t=\sum_{k=a}^{b-1} f(k), \quad a \leq b ;
\end{gathered}
$$

(3) $\mathbb{T}=\left\{2^{n}, n \in \mathbb{N}_{0}\right\}$,

$$
\begin{gathered}
\sigma(t)=2 t, \quad \rho(t)=\frac{t}{2}, \quad f^{\Delta}(t)=\frac{f(2 t)-f(t)}{t}, \\
\int_{a}^{b} f(t) \Delta t=\sum_{k=\log _{2} a}^{\log _{2} b-1} f\left(2^{k}\right) 2^{k}, \quad a \leq b .
\end{gathered}
$$

Definition 1. A solution $x$ of (12) is said to have a generalized zero at $t^{*} \in \mathbb{T}$ if $x\left(t^{*}\right) x\left(\sigma\left(t^{*}\right)\right) \leq 0$, and it is said to be nonoscillatory on $\mathbb{T}$ if there exists $t_{0} \in \mathbb{T}$ such that $x(t) x(\sigma(t))>0$ for all $t>t_{0}$. Otherwise, it is oscillatory. Equation (12) is said to be oscillatory if all solutions of (12) are oscillatory.

For simplicity, throughout this paper, we denote $(a, b) \cap$ $\mathbb{T}=(a, b)_{\mathbb{T}}$, where $a, b \in \mathbb{R}$, and $[a, b]_{\mathbb{T}},[a, b)_{\mathbb{T}},(a, b]_{\mathbb{T}}$ are denoted similarly.

To establish new oscillation criteria of (12), we give three lemmas in this section.

Lemma 2. Assume that (C1)-(C4) hold, and there exists a sufficiently large $t_{1} \in\left[t_{0}, \infty\right)_{\mathbb{T}}$ such that $x(t)$ is a solution of (12) satisfying $x(t)>0$ for $t \in\left[t_{1}, \infty\right)_{\mathbb{T}}$. Then for $t \in\left[t_{1}, \infty\right)_{\mathbb{T}}$, we have

$$
x^{\Delta}(t)>0, \quad\left[\frac{r(t)\left(x^{\Delta}(t)\right)^{\gamma}}{e_{-p / r}\left(t, t_{1}\right)}\right]^{\Delta}<0 .
$$


Proof. Let $t_{1} \in\left[t_{0}, \infty\right)_{\mathbb{T}}$ such that $x(t)$ is a solution of (12) satisfying $x(t)>0$ for $t \in\left[t_{1}, \infty\right)_{\mathbb{\pi}}$; then we also have $x(g(t))>0$. It is obvious that (12) is equivalent to

$$
\begin{aligned}
& \frac{\left(r(t) \varphi_{\gamma}\left(x^{\Delta}(t)\right)\right)^{\Delta} e_{-p / r}\left(t, t_{1}\right)+p(t) \varphi_{\gamma}\left(x^{\Delta}(t)\right) e_{-p / r}\left(t, t_{1}\right)}{e_{-p / r}\left(t, t_{1}\right) e_{-p / r}\left(\sigma(t), t_{1}\right)} \\
& +\frac{f(t, x(g(t)))}{e_{-p / r}\left(\sigma(t), t_{1}\right)}=0,
\end{aligned}
$$

which is simplified as

$$
\left[\frac{r(t) \varphi_{\gamma}\left(x^{\Delta}(t)\right)}{e_{-p / r}\left(t, t_{1}\right)}\right]^{\Delta}+\frac{f(t, x(g(t)))}{e_{-p / r}\left(\sigma(t), t_{1}\right)}=0 .
$$

By (12) and (C4), we have

$$
\left[\frac{r(t) \varphi_{\gamma}\left(x^{\Delta}(t)\right)}{e_{-p / r}\left(t, t_{1}\right)}\right]^{\Delta}=-\frac{f(t, x(g(t)))}{e_{-p / r}\left(\sigma(t), t_{1}\right)}<0, \quad t \in\left[t_{1}, \infty\right)_{\mathbb{T}} .
$$

We claim that $x^{\Delta}(t)>0$ for $t \in\left[t_{1}, \infty\right)_{\mathbb{T}}$. Assume the contrary; that is, there exists $t_{2} \in\left[t_{1}, \infty\right)_{\mathbb{T}}$ such that $x^{\Delta}\left(t_{2}\right)<$ 0 . Also, for $t \in\left[t_{2}, \infty\right)_{\mathbb{T}}$ we have

$$
\left[\frac{r(t) \varphi_{\gamma}\left(x^{\Delta}(t)\right)}{e_{-p / r}\left(t, t_{2}\right)}\right]^{\Delta}<0 .
$$

Integrating (22) from $t_{2}$ to $t \in\left[\sigma\left(t_{2}\right), \infty\right)_{\mathbb{T}}$, we obtain

$$
\begin{aligned}
& \frac{r(t) \varphi_{\gamma}\left(x^{\Delta}(t)\right)}{e_{-p / r}\left(t, t_{2}\right)}-\frac{r\left(t_{2}\right) \varphi_{\gamma}\left(x^{\Delta}\left(t_{2}\right)\right)}{e_{-p / r}\left(t_{2}, t_{2}\right)} \\
& =\frac{r(t) \varphi_{\gamma}\left(x^{\Delta}(t)\right)}{e_{-p / r}\left(t, t_{2}\right)}-r\left(t_{2}\right) \varphi_{\gamma}\left(x^{\Delta}\left(t_{2}\right)\right)<0 .
\end{aligned}
$$

That is,

$$
r(t) \varphi_{\gamma}\left(x^{\Delta}(t)\right)<r\left(t_{2}\right) \varphi_{\gamma}\left(x^{\Delta}\left(t_{2}\right)\right) e_{-p / r}\left(t, t_{2}\right),
$$

which implies that

$$
x^{\Delta}(t)<r^{1 / \gamma}\left(t_{2}\right) x^{\Delta}\left(t_{2}\right)\left(\frac{e_{-p / r}\left(t, t_{2}\right)}{r(t)}\right)^{1 / \gamma} .
$$

Integrating (25) from $t_{2}$ to $t \in\left[\sigma\left(t_{2}\right), \infty\right)_{\mathbb{T}}$ and letting $t \rightarrow$ $\infty$, by $(\mathrm{C} 2)$ we obtain

$x(t)$

$$
<x\left(t_{2}\right)+r^{1 / \gamma}\left(t_{2}\right) x^{\Delta}\left(t_{2}\right) \int_{t_{2}}^{t}\left(\frac{e_{-p / r}\left(s, t_{2}\right)}{r(s)}\right)^{1 / \gamma} \Delta s \longrightarrow-\infty,
$$

which contradicts $x(t)>0$. Hence $x^{\Delta}(t)>0$ for $t \in\left[t_{1}, \infty\right)_{\mathbb{T}}$, and $\varphi_{\gamma}\left(x^{\Delta}(t)\right)=\left(x^{\Delta}(t)\right)^{\gamma}$. By $(21)$, we obtain

$$
\left[\frac{r(t)\left(x^{\Delta}(t)\right)^{\gamma}}{e_{-p / r}\left(t, t_{1}\right)}\right]^{\Delta}<0 .
$$

\section{Lemma 2 is proved.}

Lemma 3. Assume that (C1)-(C4) hold and $x(t)$ is a solution of (12) satisfying $x(t)>0$ for $t \in\left[t_{1}, \infty\right)_{\mathbb{T}}$ with $t_{1} \in\left[t_{0}, \infty\right)_{\mathbb{T}}$. Then for $t \in\left[t_{1}, \infty\right)_{\mathbb{T}}$, if $0<\gamma<1$, we have

$$
\left(\frac{x^{\Delta}(t)}{x^{\sigma}(t)}\right)^{1-\gamma} \geq \alpha_{1}(t)
$$

and if $\gamma \geq 1$, we have

$$
\left(\frac{x(t)}{x^{\Delta}(t)}\right)^{\gamma-1} \geq \alpha_{2}(t)
$$

where

$$
\begin{gathered}
\alpha_{1}(t)=\left(\frac{e_{-p / r}\left(t, t_{1}\right)}{r(t)} \int_{t}^{\infty} \frac{q(s)}{e_{-p / r}\left(\sigma(s), t_{1}\right)} \Delta s\right)^{(1-\gamma) / \gamma}, \\
\alpha_{2}(t)=\left(\left(\frac{r(t)}{e_{-p / r}\left(t, t_{1}\right)}\right)^{1 / \gamma} \int_{t_{1}}^{t}\left(\frac{e_{-p / r}\left(s, t_{1}\right)}{r(s)}\right)^{1 / \gamma} \Delta s\right)^{\gamma-1} .
\end{gathered}
$$

Proof. Since $x(t)$ is a solution of (12) satisfying $x(t)>0$ for $t \in\left[t_{1}, \infty\right)_{\mathbb{T}}$ with $t_{1} \in \mathbb{T}$, by Lemma 2 we have

$$
x^{\Delta}(t)>0, \quad\left[\frac{r(t)\left(x^{\Delta}(t)\right)^{\gamma}}{e_{-p / r}\left(t, t_{1}\right)}\right]^{\Delta}<0, \quad t \in\left[t_{1}, \infty\right)_{\mathbb{T}} .
$$

From (21) it follows that

$$
\left[\frac{r(t)\left(x^{\Delta}(t)\right)^{\gamma}}{e_{-p / r}\left(t, t_{1}\right)}\right]^{\Delta}=-\frac{f(t, x(g(t)))}{e_{-p / r}\left(\sigma(t), t_{1}\right)} .
$$

Integrating (32) from $\infty$ to $t$ with $t \in \mathbb{T}$, we obtain

$$
\begin{aligned}
& \frac{r(t)\left(x^{\Delta}(t)\right)^{\gamma}}{e_{-p / r}\left(t, t_{1}\right)} \\
& \geq \int_{\infty}^{t}\left[\frac{r(s)\left(x^{\Delta}(s)\right)^{\gamma}}{e_{-p / r}\left(s, t_{1}\right)}\right]^{\Delta} \Delta s=\int_{t}^{\infty} \frac{f(s, x(g(s)))}{e_{-p / r}\left(\sigma(s), t_{1}\right)} \Delta s \\
& \geq \int_{t}^{\infty} \frac{q(s) x^{\gamma}(g(s))}{e_{-p / r}\left(\sigma(s), t_{1}\right)} \Delta s \\
& \geq x^{\gamma}(g(t)) \int_{t}^{\infty} \frac{q(s)}{e_{-p / r}\left(\sigma(s), t_{1}\right)} \Delta s \\
& \geq\left(x^{\sigma}(t)\right)^{\gamma} \int_{t}^{\infty} \frac{q(s)}{e_{-p / r}\left(\sigma(s), t_{1}\right)} \Delta s .
\end{aligned}
$$


Hence when $0<\gamma<1$, we have

$$
\left(\frac{x^{\Delta}(t)}{x^{\sigma}(t)}\right)^{1-\gamma} \geq \alpha_{1}(t), \quad t \in\left[t_{1}, \infty\right)_{\mathbb{T}}
$$

Since $\left[r(t)\left(x^{\Delta}(t)\right)^{\gamma} / e_{-p / r}\left(t, t_{1}\right)\right]^{\Delta}<0, t \in\left[t_{1}, \infty\right)_{\mathbb{T}}$, for $\gamma \geq 1$, we get

$$
\begin{aligned}
x(t) & \geq x(t)-x\left(t_{1}\right)=\int_{t_{1}}^{t} \frac{\left(r(s)\left(x^{\Delta}(s)\right)^{\gamma} / e_{-p / r}\left(s, t_{1}\right)\right)^{1 / \gamma}}{\left(r(s) / e_{-p / r}\left(s, t_{1}\right)\right)^{1 / \gamma}} \Delta s \\
& \geq\left(\frac{r(t)\left(x^{\Delta}(t)\right)^{\gamma}}{e_{-p / r}\left(t, t_{1}\right)}\right)^{1 / \gamma} \int_{t_{1}}^{t}\left(\frac{e_{-p / r}\left(s, t_{1}\right)}{r(s)}\right)^{1 / \gamma} \Delta s,
\end{aligned}
$$

and we obtain

$$
\left(\frac{x(t)}{x^{\Delta}(t)}\right)^{\gamma-1} \geq \alpha_{2}(t), \quad t \in\left[t_{1}, \infty\right)_{\mathbb{T}} .
$$

Lemma 3 is proved.

Remark 4. In Lemma 3, when $0<\gamma<1$ and for any $t \in$ $\left[t_{1}, \infty\right)_{\mathbb{T}}$, the integral $\int_{t}^{\infty} q(s) \Delta s$ in $\alpha_{1}(t)$ must be convergent, which means

$$
\int_{t}^{\infty} q(s) \Delta s<\infty
$$

Lemma 5. Assume that (C1)-(C4) hold and $x(t)$ is a solution of (12) satisfying $x(t)>0$ for $t \in\left[t_{1}, \infty\right)_{\mathbb{T}}$ with $t_{1} \in\left[t_{0}, \infty\right)_{\mathbb{T}}$. For $t \in\left[t_{1}, \infty\right)_{\mathbb{T}}$, define

$$
u(t)=A(t) \frac{r(t)\left(x^{\Delta}(t)\right)^{\gamma}}{e_{-p / r}\left(t, t_{1}\right) x^{\gamma}(t)}+B(t)
$$

where $A \in C_{r d}^{1}(\mathbb{T},(0, \infty)), B \in C_{r d}^{1}(\mathbb{T}, \mathbb{R})$. Then $u(t)$ satisfies

$$
\begin{gathered}
u^{\Delta}(t)+\Phi_{0}(t)-A^{\Delta}(t)\left(\frac{u(t)-B(t)}{A(t)}\right)^{\sigma} \\
+\frac{\gamma A(t) \alpha(t)}{r(t)}\left[\left(\frac{u(t)-B(t)}{A(t)}\right)^{\sigma}\right]^{2} \leq 0,
\end{gathered}
$$

where

$$
\begin{aligned}
\Phi_{0}(t) & =\frac{A(t) q(t)}{e_{-p / t}\left(\sigma(t), t_{1}\right)}-B^{\Delta}(t), \\
\alpha(t) & = \begin{cases}\alpha_{1}(t), & 0<\gamma<1, \\
\alpha_{2}(t), & \gamma \geq 1 .\end{cases}
\end{aligned}
$$

Proof. Without loss of generality we may assume there exists $t_{1} \in\left[t_{0}, \infty\right)_{\mathbb{T}}$ such that $x(t)>0$ for $t \in\left[t_{1}, \infty\right)_{\mathbb{T}}$; then
Lemmas 2 and 3 hold. Let $u(t)$ be defined by (38). Then, differentiating (38) and using (12), it follows that

$$
\begin{aligned}
u^{\Delta}= & \left(\frac{A}{x^{\gamma}}\right)\left(\frac{r\left(x^{\Delta}\right)^{\gamma}}{e_{-p / r}}\right)^{\Delta}+\left(\frac{A}{x^{\gamma}}\right)^{\Delta}\left(\frac{r\left(x^{\Delta}\right)^{\gamma}}{e_{-p / r}}\right)^{\sigma}+B^{\Delta} \\
= & -\frac{A}{x^{\gamma}} \frac{f(t, x(g(t)))}{e_{-p / r}^{\sigma}}+\frac{A^{\Delta} x^{\gamma}-A\left(x^{\gamma}\right)^{\Delta}}{x^{\gamma}\left(x^{\gamma}\right)^{\sigma}}\left(\frac{r\left(x^{\Delta}\right)^{\gamma}}{e_{-p / r}}\right)^{\sigma} \\
& +B^{\Delta} .
\end{aligned}
$$

Using the fact that $f(t, x(g(t))) \geq q(t) x^{\gamma}(g(t)) \geq q(t) x^{\gamma}(t)$, we obtain

$u^{\Delta}$

$$
\begin{aligned}
& \leq-\frac{A q}{e_{-p / r}^{\sigma}}+B^{\Delta}+A^{\Delta}\left(\frac{r\left(x^{\Delta}\right)^{\gamma}}{e_{-p / r} x^{\gamma}}\right)^{\sigma}-A \frac{\left(x^{\gamma}\right)^{\Delta}}{x^{\gamma}}\left(\frac{r\left(x^{\Delta}\right)^{\gamma}}{e_{-p / r} x^{\gamma}}\right)^{\sigma} \\
& =-\frac{A q}{e_{-p / r}^{\sigma}}+B^{\Delta}+A^{\Delta}\left(\frac{u-B}{A}\right)^{\sigma}-A \frac{\left(x^{\gamma}\right)^{\Delta}}{x^{\gamma}}\left(\frac{u-B}{A}\right)^{\sigma} .
\end{aligned}
$$

When $0<\gamma<1$, using the Pötzsche chain rule, we have

$$
\left(x^{\gamma}\right)^{\Delta}=\gamma \int_{0}^{1}\left(x+h \mu x^{\Delta}\right)^{\gamma-1} d h \cdot x^{\Delta} \geq \gamma\left(x^{\sigma}\right)^{\gamma-1} x^{\Delta},
$$

and it follows that

$$
\frac{\left(x^{\gamma}\right)^{\Delta}}{x^{\gamma}} \geq \frac{\gamma\left(x^{\sigma}\right)^{\gamma-1} x^{\Delta}}{x^{\gamma}}=\gamma \frac{x^{\Delta}}{x^{\sigma}}\left(\frac{x^{\sigma}}{x}\right)^{\gamma}
$$

By Lemmas 2 and 3 , for $t \in\left[t_{1}, \infty\right)_{\mathbb{T}}$, we obtain

$$
\begin{aligned}
& \frac{x^{\Delta}}{x^{\sigma}}=\frac{1}{r} \frac{r\left(x^{\Delta}\right)^{\gamma}}{\left(x^{\sigma}\right)^{\gamma}}\left(\frac{x^{\Delta}}{x^{\sigma}}\right)^{1-\gamma} \\
& \geq \frac{\alpha_{1}}{r} \frac{\left(r\left(x^{\Delta}\right)^{\gamma}\right)^{\sigma}}{\left(x^{\gamma}\right)^{\sigma}}=\frac{\alpha_{1}}{r}\left(\frac{u-B}{A}\right)^{\sigma}, \\
& \frac{x^{\sigma}}{x} \geq 1 .
\end{aligned}
$$

So (42) becomes

$$
u^{\Delta} \leq-\frac{A q}{e_{-p / r}^{\sigma}}+B^{\Delta}+A^{\Delta}\left(\frac{u-B}{A}\right)^{\sigma}-\frac{\gamma A \alpha_{1}}{r}\left[\left(\frac{u-B}{A}\right)^{\sigma}\right]^{2} .
$$

When $\gamma \geq 1$, we have

$$
\left(x^{\gamma}\right)^{\Delta}=\gamma \int_{0}^{1}\left(x+h \mu x^{\Delta}\right)^{\gamma-1} d h \cdot x^{\Delta} \geq \gamma x^{\gamma-1} x^{\Delta},
$$


and it follows that

$$
\frac{\left(x^{\gamma}\right)^{\Delta}}{x^{\gamma}} \geq \frac{\gamma x^{\gamma-1} x^{\Delta}}{x^{\gamma}}=\frac{\gamma x^{\Delta}}{x}
$$

By Lemmas 2 and 3 , for $t \in\left[t_{1}, \infty\right)_{\mathbb{T}}$, we obtain

$$
\frac{x^{\Delta}}{x}=\frac{1}{r} \frac{r\left(x^{\Delta}\right)^{\gamma}}{x^{\gamma}}\left(\frac{x}{x^{\Delta}}\right)^{\gamma-1} \geq \frac{\alpha_{2}}{r} \frac{\left(r\left(x^{\Delta}\right)^{\gamma}\right)^{\sigma}}{\left(x^{\gamma}\right)^{\sigma}}=\frac{\alpha_{2}}{r}\left(\frac{u-B}{A}\right)^{\sigma} .
$$

So (42) becomes

$$
u^{\Delta} \leq-\frac{A q}{e_{-p / r}^{\sigma}}+B^{\Delta}+A^{\Delta}\left(\frac{u-B}{A}\right)^{\sigma}-\frac{\gamma A \alpha_{2}}{r}\left[\left(\frac{u-B}{A}\right)^{\sigma}\right]^{2} .
$$

By (46) and (50), (39) holds. Lemma 5 is proved.

\section{Main Results}

In this section, we will derive new oscillation criteria of (12). Our approach to oscillation results of (12) is based on the application of the generalized Riccati transformation. Firstly, we give some definitions.

Let $D_{0}=\{s \in \mathbb{T}: s \geq 0\}$ and $D=\left\{(t, s) \in \mathbb{T}^{2}: t \geq s \geq 0\right\}$. For any function $f(t, s): \mathbb{T}^{2} \rightarrow \mathbb{R}$, denote by $f_{2}^{\Delta}$ the partial derivative of $f$ with respect to $s$. Define

$$
\begin{aligned}
(\mathscr{A}, \mathscr{B})=\left\{(A, B): A(s) \in C_{r d}^{1}\left(D_{0},(0, \infty)\right),\right. & \left.B(s) \in C_{r d}^{1}\left(D_{0}, \mathbb{R}\right), s \in D_{0}\right\} ; \\
\mathscr{H}=\{ & H(t, s) \in C^{1}(D,[0, \infty)): H(t, t)=0, \\
& \left.H(t, s)>0, H_{2}^{\Delta}(t, s) \leq 0, t>s \geq 0\right\} .
\end{aligned}
$$

These function classes will be used throughout this paper. Now, we are in a position to give our first theorem.

Theorem 6. Assume that (C1)-(C4) hold and that there exist $(A, B) \in(\mathscr{A}, \mathscr{B})$ and $H \in \mathscr{H}$ such that, for any $t_{1} \in \mathbb{T}$,

$$
\begin{aligned}
\limsup _{t \rightarrow \infty} & \frac{1}{H\left(t, t_{1}\right)} \\
\times \int_{t_{1}}^{t} & {\left[H(t, s) \Phi_{0}(s)-H_{2}^{\Delta}(t, s) B^{\sigma}(s)\right.} \\
& \left.\quad-\frac{r(s)\left(H_{2}^{\Delta}(t, s) A^{\sigma}(s)+H(t, s) A^{\Delta}(s)\right)^{2}}{4 \gamma H(t, s) A(s) \alpha(s)}\right] \Delta s=\infty,
\end{aligned}
$$

where $\Phi_{0}$ and $\alpha$ are defined as before. Then, (12) is oscillatory.

Proof. Assume that (12) is not oscillatory. Without loss of generality we may assume there exists $t_{1} \in\left[t_{0}, \infty\right)_{\mathbb{T}}$ such that $x(t)>0$ for $t \in\left[t_{1}, \infty\right)_{\mathbb{T}}$. Let $u(t)$ be defined by (38). Then by Lemma 5, (39) holds.

For simplicity in the following, we let $H_{\sigma}=H(t, \sigma(s))$, $H=H(t, s), H_{2}^{\Delta}=H_{2}^{\Delta}(t, s)$, and omit the arguments in the integrals.

Multiplying (39), where $t$ is replaced by $s$, by $H$, and integrating it with respect to $s$ from $t_{1}$ to $t \in\left[\sigma\left(t_{1}\right), \infty\right)_{\mathbb{V}}$, we obtain

$$
\begin{aligned}
& \int_{t_{1}}^{t} H \Phi_{0} \Delta s \\
& \leq-\int_{t_{1}}^{t} H\left(u^{\Delta}-A^{\Delta}\left(\frac{u-B}{A}\right)^{\sigma}+\frac{\gamma A \alpha}{r}\left[\left(\frac{u-B}{A}\right)^{\sigma}\right]^{2}\right) \Delta s .
\end{aligned}
$$

Noting that $H(t, t)=0$, by the integration by parts formula we have

$$
\begin{aligned}
& \int_{t_{1}}^{t} H \Phi_{0} \Delta s \\
& \leq H\left(t, t_{1}\right) u\left(t_{1}\right)+\int_{t_{1}}^{t}\left(H_{2}^{\Delta} u^{\sigma}+H A^{\Delta}\left(\frac{u-B}{A}\right)^{\sigma}\right. \\
& \left.\quad-\frac{\gamma H A \alpha}{r}\left[\left(\frac{u-B}{A}\right)^{\sigma}\right]^{2}\right) \Delta s \\
& =H\left(t, t_{1}\right) u\left(t_{1}\right)+\int_{t_{1}}^{t} H_{2}^{\Delta} B^{\sigma} \Delta s \\
& +\int_{t_{1}}^{t}\left(\left(H_{2}^{\Delta} A^{\sigma}+H A^{\Delta}\right)\left(\frac{u-B}{A}\right)^{\sigma}\right. \\
& \left.-\frac{\gamma H A \alpha}{r}\left[\left(\frac{u-B}{A}\right)^{\sigma}\right]^{2}\right) \Delta s .
\end{aligned}
$$

Since

$$
\begin{aligned}
\left(H_{2}^{\Delta} A^{\sigma}+H A^{\Delta}\right)\left(\frac{u-B}{A}\right)^{\sigma}-\frac{\gamma H A \alpha}{r}\left[\left(\frac{u-B}{A}\right)^{\sigma}\right]^{2} \\
=\frac{r\left(H_{2}^{\Delta} A^{\sigma}+H A^{\Delta}\right)^{2}}{4 \gamma H A \alpha} \\
\quad-\frac{\gamma H A \alpha}{r}\left[\left(\frac{u-B}{A}\right)^{\sigma}-\frac{r\left(H_{2}^{\Delta} A^{\sigma}+H A^{\Delta}\right)}{2 \gamma H A \alpha}\right]^{2} \\
\leq \frac{r\left(H_{2}^{\Delta} A^{\sigma}+H A^{\Delta}\right)^{2}}{4 \gamma H A \alpha}
\end{aligned}
$$


by (54) we have

$$
\begin{aligned}
& \int_{t_{1}}^{t} H \Phi_{0} \Delta s \\
& \quad \leq H\left(t, t_{1}\right) u\left(t_{1}\right)+\int_{t_{1}}^{t}\left(H_{2}^{\Delta} B^{\sigma}+\frac{r\left(H_{2}^{\Delta} A^{\sigma}+H A^{\Delta}\right)^{2}}{4 \gamma H A \alpha}\right) \Delta s .
\end{aligned}
$$

Hence

$$
\begin{aligned}
& \frac{1}{H\left(t, t_{1}\right)} \int_{t_{1}}^{t}\left[H \Phi_{0}-H_{2}^{\Delta} B^{\sigma}-\frac{r\left(H_{2}^{\Delta} A^{\sigma}+H A^{\Delta}\right)^{2}}{4 \gamma H A \alpha}\right] \Delta s \\
& \quad \leq u\left(t_{1}\right)<\infty
\end{aligned}
$$

which contradicts (52) and completes the proof.

When $B=0$, (38) is simplified as

$$
u(t)=A(t) \frac{r(t)\left(x^{\Delta}(t)\right)^{\gamma}}{e_{-p / r}\left(t, t_{1}\right) x^{\gamma}(t)}, \quad t \in\left[t_{1}, \infty\right)_{\mathbb{T}} .
$$

Now we have the following theorem.

Theorem 7. Assume that (C1)-(C4) hold and that there exists $A \in C_{r d}^{1}\left(D_{0},(0, \infty)\right)$ such that, for any $t_{1} \in \mathbb{T}$,

$$
\limsup _{t \rightarrow \infty} \int_{t_{1}}^{t}\left[\frac{A(s) q(s)}{e_{-p / r}\left(\sigma(s), t_{1}\right)}-\frac{r(s)\left(A^{\Delta}(s)\right)^{2}}{4 \gamma A(s) \alpha(s)}\right] \Delta s=\infty \text {, }
$$

where $\alpha$ is defined as before. Then, (12) is oscillatory.

Proof. Assume that (12) is not oscillatory. Without loss of generality we may assume there exists $t_{1} \in\left[t_{0}, \infty\right)_{\mathbb{T}}$ such that $x(t)>0$ for $t \in\left[t_{1}, \infty\right)_{\mathbb{T}}$. Let $u(t)$ be defined by (58). Then by Lemma 5, we have

$$
\begin{gathered}
u^{\Delta}(t)+\frac{A(t) q(t)}{e_{-p / r}\left(\sigma(t), t_{1}\right)}-A^{\Delta}(t)\left(\frac{u(t)}{A(t)}\right)^{\sigma} \\
+\frac{\gamma A(t) \alpha(t)}{r(t)}\left[\left(\frac{u(t)}{A(t)}\right)^{\sigma}\right]^{2} \leq 0,
\end{gathered}
$$

which implies that

$$
\begin{aligned}
u^{\Delta} & \leq-\frac{A q}{e_{-p / r}^{\sigma}}+A^{\Delta}\left(\frac{u}{A}\right)^{\sigma}-\frac{\gamma A \alpha}{r}\left[\left(\frac{u}{A}\right)^{\sigma}\right]^{2} \\
& =-\frac{A q}{e_{-p / r}^{\sigma}}+\frac{r\left(A^{\Delta}\right)^{2}}{4 \gamma A \alpha}-\frac{\gamma A \alpha}{r}\left[\left(\frac{u}{A}\right)^{\sigma}-\frac{A^{\Delta} r}{2 \gamma A \alpha}\right]^{2} \\
& \leq-\frac{A q}{e_{-p / r}^{\sigma}}+\frac{r\left(A^{\Delta}\right)^{2}}{4 \gamma A \alpha} .
\end{aligned}
$$

So we obtain

$$
\frac{A(t) q(t)}{e_{-p / r}\left(\sigma(t), t_{1}\right)}-\frac{r(t)\left(A^{\Delta}(t)\right)^{2}}{4 \gamma A(t) \alpha(t)} \leq-u^{\Delta}(t) .
$$

Letting $t$ be replaced by $s$, and integrating (62) with respect to $s$ from $t_{1}$ to $t \in\left[\sigma\left(t_{1}\right), \infty\right)_{\mathbb{T}}$, we obtain

$$
\begin{aligned}
& \int_{t_{1}}^{t}\left[\frac{A(s) q(s)}{e_{-p / r}\left(\sigma(s), t_{1}\right)}-\frac{r(s)\left(A^{\Delta}(s)\right)^{2}}{4 \gamma A(s) \alpha(s)}\right] \Delta s \\
& \quad \leq-\int_{t_{1}}^{t} u^{\Delta}(s) \Delta s=u\left(t_{1}\right)-u(t)<u\left(t_{1}\right)<\infty,
\end{aligned}
$$

which contradicts (59) and completes the proof.

When $p \geq 0$, it is easy to obtain $-p / r \leq 0$, so we have

$$
\frac{1}{e_{-p / r}\left(\sigma(t), t_{1}\right)} \geq 1 \text {. }
$$

Then, Theorems 6 and 7 can be simplified as the following corollaries, respectively.

Corollary 8. Assume that (C1)-(C4) hold and that there exist $(A, B) \in(\mathscr{A}, \mathscr{B})$ and $H \in \mathscr{H}$ such that, for any $t_{1} \in \mathbb{T}$,

$$
\begin{aligned}
\limsup _{t \rightarrow \infty} & \frac{1}{H\left(t, t_{1}\right)} \\
\times \int_{t_{1}}^{t}[ & H(t, s)\left(A(s) q(s)-B^{\Delta}(s)\right)-H_{2}^{\Delta}(t, s) B^{\sigma}(s) \\
& \left.\quad-\frac{r(s)\left(H_{2}^{\Delta}(t, s) A^{\sigma}(s)+H(t, s) A^{\Delta}(s)\right)^{2}}{4 \gamma H(t, s) A(s) \alpha(s)}\right] \Delta s=\infty .
\end{aligned}
$$

Then, (12) is oscillatory.

Corollary 9. Assume that (C1)-(C4) hold and that there exists $A \in C_{r d}^{1}\left(D_{0},(0, \infty)\right)$ such that, for any $t_{1} \in \mathbb{T}$,

$$
\limsup _{t \rightarrow \infty} \int_{t_{1}}^{t}\left[A(s) q(s)-\frac{r(s)\left(A^{\Delta}(s)\right)^{2}}{4 \gamma A(s) \alpha(s)}\right] \Delta s=\infty .
$$

Then, (12) is oscillatory.

Remark 10. Compared to the theorems and corollaries in [15], the conclusions of Theorems 6 and 7 and Corollaries 8 and 9 are much simpler, and the proofs of Theorems 6 and 7 are more convenient. Furthermore, $p(t)$ may change sign in Theorems 6 and 7, for the employment of the function $e_{-p / r}\left(t, t_{1}\right)$

Remark 11. Consider the equations as the following form:

$$
\left(r(t) \varphi_{\gamma}\left(x^{\Delta}(t)\right)\right)^{\Delta}+p(t) \varphi_{\gamma}\left(x^{\Delta \sigma}(t)\right)+f(t, x(g(t)))=0 .
$$


It is obvious that (67) is equivalent to

$$
\begin{aligned}
& \left(r(t) \varphi_{\gamma}\left(x^{\Delta}(t)\right)\right)^{\Delta} e_{p / r^{\sigma}}\left(t, t_{1}\right)+p(t) \varphi_{\gamma}\left(x^{\Delta}(t)\right) e_{p / r^{\sigma}}\left(t, t_{1}\right) \\
& \quad+f(t, x(g(t))) e_{p / r^{\sigma}}\left(t, t_{1}\right)=0,
\end{aligned}
$$

which is simplified as

$$
\left(r(t) e_{p / r^{\sigma}}\left(t, t_{1}\right) \varphi_{\gamma}\left(x^{\Delta}(t)\right)\right)^{\Delta}+f(t, x(g(t))) e_{p / r^{\sigma}}\left(t, t_{1}\right)=0 .
$$

We can get the similar oscillation results as before, where

$$
\frac{r(t)}{e_{-p / r}\left(t, t_{1}\right)}, \quad \frac{q(t)}{e_{-p / r}\left(\sigma(t), t_{1}\right)}
$$

are replaced by

$$
r(t) e_{p / r^{\sigma}}\left(t, t_{1}\right), \quad q(t) e_{p / r^{\sigma}}\left(t, t_{1}\right),
$$

respectively, in Lemmas 2, 3, and 5, Theorems 6 and 7, and Corollaries 8 and 9.

\section{Examples}

In this section, we will show the application of our oscillation results in two examples. Firstly, we give an example to demonstrate Theorem 6 .

Example 12. Consider the equation

$$
\left(t \varphi_{\gamma}\left(x^{\Delta}(t)\right)\right)^{\Delta}-\varphi_{\gamma}\left(x^{\Delta}(t)\right)+\left(t+\frac{1}{t}\right) x^{\gamma}(g(t))=0,
$$

where $r(t)=t, p(t)=-1, q(t)=1 / t, \gamma>2$, so we have $-p(t) / r(t)=1 / t$. Letting $H(t, s)=(t-s)^{2},(A, B)=\left(s^{2}, 1\right)$, we have the following results.

(i) $\mathbb{T}=\mathbb{N}$. In this case, there exists $t_{1} \in[1, \infty)_{\mathbb{T}}$ such that

$$
\begin{aligned}
& e_{-p / r}\left(t, t_{0}\right)=e_{1 / t}(t, 1)=\exp \left(\int_{1}^{t} \xi_{1}\left(\frac{1}{\tau}\right) \Delta \tau\right) \\
&=\exp \left(\int_{1}^{t} \log \left(1+\frac{1}{\tau}\right) \Delta \tau\right)=\prod_{k=1}^{t-1}\left(1+\frac{1}{k}\right) \\
&=t \\
& e_{-p / r}\left(t, t_{1}\right)=e_{1 / t}\left(t, t_{1}\right)=\exp \left(\int_{t_{1}}^{t} \xi_{1}\left(\frac{1}{\tau}\right) \Delta \tau\right) \\
&=\exp \left(\int_{t_{1}}^{t} \log \left(1+\frac{1}{\tau}\right) \Delta \tau\right)=\prod_{k=t_{1}}^{t-1}\left(1+\frac{1}{k}\right) \\
&=\frac{t}{t_{1}}, \\
& \int_{t_{0}}^{\infty}\left(\frac{e_{-p / r}\left(t, t_{0}\right)}{r(t)}\right)^{1 / \gamma} \Delta t=\int_{1}^{\infty} \Delta t=\infty,
\end{aligned}
$$

$$
\begin{aligned}
\alpha_{2}(t) & =\left(\left(\frac{r(t)}{e_{-p / r}\left(t, t_{1}\right)}\right)^{1 / \gamma} \int_{t_{1}}^{t}\left(\frac{e_{-p / r}\left(s, t_{1}\right)}{r(s)}\right)^{1 / \gamma} \Delta s\right)^{\gamma-1} \\
& =\left(t_{1}^{1 / \gamma} \int_{t_{1}}^{t}\left(\frac{1}{t_{1}}\right)^{1 / \gamma} \Delta s\right)^{\gamma-1}=\left(\int_{t_{1}}^{t} \Delta s\right)^{\gamma-1} \\
& =\left(t-t_{1}\right)^{\gamma-1} .
\end{aligned}
$$

Hence

$$
\begin{aligned}
& \limsup _{t \rightarrow \infty} \frac{1}{H\left(t, t_{1}\right)} \\
& \times \int_{t_{1}}^{t}\left[H(t, s) \Phi_{0}(s)-H_{2}^{\Delta}(t, s) B^{\sigma}(s)\right. \\
& \left.\quad-\frac{r(s)\left(H_{2}^{\Delta}(t, s) A^{\sigma}(s)+H(t, s) A^{\Delta}(s)\right)^{2}}{4 \gamma H(t, s) A(s) \alpha(s)}\right] \Delta s \\
& \left.=\limsup _{t \rightarrow \infty} \frac{1}{\left(t-t_{1}\right)^{2}}\right] \Delta s \\
& \left.\times \int_{t_{1}}^{t}\left[\frac{(t-s)^{2} s^{2} \cdot s^{-1}}{(s+1) \cdot t_{1}^{-1}+(2 t-2 s-1)}\right] \Delta\right)^{2} \\
& -\frac{s\left(-(2 t-2 s-1)(s+1)^{2}+(t-s)^{2}(2 s+1)\right.}{4 \gamma(t-s)^{2} s^{2}\left(s-t_{1}\right)^{\gamma-1}}
\end{aligned}
$$

$=\infty$.

That is, (52) holds. By Theorem 6 we see that (72) is oscillatory.

(ii) $\mathbb{T}=\left\{2^{n}, n \in \mathbb{N}_{0}\right\}$. In this case, there exists $t_{1} \in[1, \infty)_{\mathbb{T}}$ such that

$$
\begin{aligned}
e_{-p / r}\left(t, t_{0}\right) & =e_{1 / t}(t, 1)=\exp \left(\int_{1}^{t} \xi_{\tau}\left(\frac{1}{\tau}\right) \Delta \tau\right) \\
& =\exp \left(\int_{1}^{t} \frac{1}{\tau} \log \left(1+\tau \cdot \frac{1}{\tau}\right) \Delta \tau\right) \\
& =\exp \left(\log 2 \int_{1}^{t} \frac{1}{\tau} \Delta \tau\right)=2^{\log _{2} t}=t, \\
e_{-p / r}\left(t, t_{1}\right) & =e_{1 / t}\left(t, t_{1}\right)=\exp \left(\int_{t_{1}}^{t} \xi_{\tau}\left(\frac{1}{\tau}\right) \Delta \tau\right) \\
& =\exp \left(\int_{t_{1}}^{t} \frac{1}{\tau} \log \left(1+\tau \cdot \frac{1}{\tau}\right) \Delta \tau\right) \\
& =\exp \left(\log 2 \int_{t_{1}}^{t} \frac{1}{\tau} \Delta \tau\right)=2^{\log _{2} t / t_{1}}=\frac{t}{t_{1}},
\end{aligned}
$$




$$
\begin{aligned}
& \int_{t_{0}}^{\infty}\left(\frac{e_{-p / r}\left(t, t_{0}\right)}{r(t)}\right)^{1 / \gamma} \Delta t=\int_{1}^{\infty} \Delta t=\infty \\
\alpha_{2}(t)= & \left(\left(\frac{r(t)}{e_{-p / r}\left(t, t_{1}\right)}\right)^{1 / \gamma} \int_{t_{1}}^{t}\left(\frac{e_{-p / r}\left(s, t_{1}\right)}{r(s)}\right)^{1 / \gamma} \Delta s\right)^{\gamma-1} \\
= & \left(t_{1}^{1 / \gamma} \int_{t_{1}}^{t}\left(\frac{1}{t_{1}}\right)^{1 / \gamma} \Delta s\right)^{\gamma-1}=\left(\int_{t_{1}}^{t} \Delta s\right)^{\gamma-1} \\
= & \left(t-t_{1}\right)^{\gamma-1} .
\end{aligned}
$$

Hence

$$
\begin{aligned}
& \limsup _{t \rightarrow \infty} \frac{1}{H\left(t, t_{1}\right)} \\
& \times \int_{t_{1}}^{t}\left[H(t, s) \Phi_{0}(s)-H_{2}^{\Delta}(t, s) B^{\sigma}(s)\right. \\
& \left.\quad-\frac{r(s)\left(H_{2}^{\Delta}(t, s) A^{\sigma}(s)+H(t, s) A^{\Delta}(s)\right)^{2}}{4 \gamma H(t, s) A(s) \alpha(s)}\right] \Delta s \\
& \left.=\limsup _{t \rightarrow \infty} \frac{1}{\left(t-t_{1}\right)^{2}}\right] \Delta s \\
& \times \int_{t_{1}}^{t}\left[\frac{(t-s)^{2} s^{2} \cdot s^{-1}}{2 s \cdot t_{1}^{-1}}+(2 t-3 s)\right. \\
& =\infty . \\
& \left.-\frac{s\left(-(2 t-3 s)(2 s)^{2}+(t-s)^{2} \cdot 3 s\right)^{2}}{4 \gamma(t-s)^{2} s^{2}\left(s-t_{1}\right)^{\gamma-1}}\right] \Delta
\end{aligned}
$$

That is, (52) holds. By Theorem 6 we see that (72) is oscillatory.

The second example illustrates Theorem 7 (or Corollary 9).

Example 13. Consider the equation

$$
\left(\frac{1}{t} \varphi_{\gamma}\left(x^{\Delta}(t)\right)\right)^{\Delta}+\frac{\lambda}{t^{2}} \varphi_{\gamma}\left(x^{\Delta}(t)\right)+\frac{1}{t^{\lambda+2}} x^{\gamma}(g(t))=0,
$$

where $r(t)=1 / t, p(t)=\lambda / t^{2}, q(t)=1 / t^{\lambda+2}=t^{-\lambda-2}, \gamma>\lambda$, where $\lambda$ is a fixed constant and satisfies $0 \leq \lambda \leq 2$, so we have $-p(t) / r(t)=-\lambda / t$. Let $H(t, s)=(t-s)^{2}, A(s)=s^{\lambda+2}$, when $\mathbb{T}=[1, \infty)$; then there exists $t_{1} \in[1, \infty)$ such that

$$
\begin{aligned}
& e_{-p / r}\left(t, t_{0}\right)=e_{-\lambda / t}(t, 1)=\exp \left(\int_{1}^{t} \xi_{0}\left(-\frac{\lambda}{\tau}\right) d \tau\right) \\
&=\exp \left(\int_{1}^{t}\left(-\frac{\lambda}{\tau}\right) d \tau\right)=\left(\frac{1}{t}\right)^{\lambda}, \\
& e_{-p / r}\left(t, t_{1}\right)=e_{-\lambda / t}\left(t, t_{1}\right)=\exp \left(\int_{t_{1}}^{t} \xi_{0}\left(-\frac{\lambda}{\tau}\right) d \tau\right) \\
&=\exp \left(\int_{t_{1}}^{t}\left(-\frac{\lambda}{\tau}\right) d \tau\right)=\left(\frac{t_{1}}{t}\right)^{\lambda}, \\
& \int_{t_{0}}^{\infty}\left(\frac{e_{-p / r}\left(t, t_{0}\right)}{r(t)}\right)^{1 / \gamma} \Delta t
\end{aligned}
$$

$$
=\int_{1}^{\infty}\left(\frac{1}{t^{\lambda-1}}\right)^{1 / \gamma} d t=\int_{1}^{\infty} \frac{1}{t^{(\lambda-1) / \gamma}} d t=\infty,
$$$$
\alpha_{1}(t)=\left(\frac{e_{-p / r}\left(t, t_{1}\right)}{r(t)} \int_{t}^{\infty} \frac{q(s)}{e_{-p / r}\left(\sigma(s), t_{1}\right)} \Delta s\right)^{(1-\gamma) / \gamma}
$$$$
=\left(\frac{t_{1}^{\lambda}}{t^{\lambda-1}} \int_{t}^{\infty} \frac{s^{-\lambda-2} \cdot s^{\lambda}}{t_{1}^{\lambda}} d s\right)^{(1-\gamma) / \gamma}
$$$$
=\left(\frac{1}{t^{\lambda-1}} \int_{t}^{\infty} \frac{1}{s^{2}} d s\right)^{(1-\gamma) / \gamma}=t^{-\lambda(1-\gamma) / \gamma}
$$

$$
\begin{aligned}
\alpha_{2}(t) & =\left(\left(\frac{r(t)}{e_{-p / r}\left(t, t_{1}\right)}\right)^{1 / \gamma} \int_{t_{1}}^{t}\left(\frac{e_{-p / r}\left(s, t_{1}\right)}{r(s)}\right)^{1 / \gamma} \Delta s\right)^{\gamma-1} \\
& =\left(\left(\frac{t^{\lambda-1}}{t_{1}^{\lambda}}\right)^{1 / \gamma} \int_{t_{1}}^{t}\left(\frac{t_{1}^{\lambda}}{s^{\lambda-1}}\right)^{1 / \gamma} d s\right)^{\gamma-1}=O\left(t^{\gamma-1}\right) .
\end{aligned}
$$

So we obtain

$$
\begin{gathered}
\limsup _{t \rightarrow \infty} \int_{t_{1}}^{t}\left[A(s) q(s)-\frac{r(s)\left(A^{\Delta}(s)\right)^{2}}{4 \gamma A(s) \alpha(s)}\right] \Delta s \\
=\underset{t \rightarrow \infty}{\limsup } \int_{t_{1}}^{t}\left[s^{\lambda+2} \cdot s^{-\lambda-2}-\frac{s^{-1}\left((\lambda+2) s^{\lambda+1}\right)^{2}}{4 \gamma s^{\lambda+2} \alpha(s)}\right] d s \\
=\underset{t \rightarrow \infty}{\limsup } \int_{t_{1}}^{t}\left[1-\frac{(\lambda+2)^{2} s^{\lambda-1}}{4 \gamma \alpha(s)}\right] d s . \\
\text { (i) } 0<\gamma<1, \alpha(s)=\alpha_{1}(s)=s^{-\lambda(1-\gamma) / \gamma} \text {. Since } \\
\lambda-1-\left(-\frac{\lambda(1-\gamma)}{\gamma}\right)=\frac{\lambda}{\gamma}-1<0,
\end{gathered}
$$

it follows that

$$
\limsup _{t \rightarrow \infty} \int_{t_{1}}^{t}\left[A(s) q(s)-\frac{r(s)\left(A^{\Delta}(s)\right)^{2}}{4 \gamma A(s) \alpha(s)}\right] \Delta s=\infty .
$$


(ii) $\gamma \geq 1, \alpha(s)=\alpha_{2}(s)=O\left(s^{\gamma-1}\right)$. Since

$$
\lambda-1-(\gamma-1)=\lambda-\gamma<0,
$$

it follows that

$$
\limsup _{t \rightarrow \infty} \int_{t_{1}}^{t}\left[A(s) q(s)-\frac{r(s)\left(A^{\Delta}(s)\right)^{2}}{4 \gamma A(s) \alpha(s)}\right] \Delta s=\infty .
$$

That is, (66) holds. By Corollary 9 we see that (77) is oscillatory.

Similarly, we can get the same conclusion when $\mathbb{T}=\{n \mid$ $n \geq 3, n \in \mathbb{N}\}$.

\section{Conflict of Interests}

The authors declare that there is no conflict of interests regarding the publication of this paper.

\section{Acknowledgment}

This project was supported by the NNSF of China (no. 11271379).

\section{References}

[1] S. Hilger, Maß kettenkalkül mit Anwendung auf Zentrumsmannigfaltigkeiten [Ph.D. thesis], Universität Würzburg, 1988.

[2] S. Hilger, "Analysis on measure chains-a unified approach to continuous and discrete calculus," Results in Mathematics, vol. 18, no. 1-2, pp. 18-56, 1990.

[3] R. P. Agarwal and M. Bohner, "Basic calculus on time scales and some of its applications," Results in Mathematics, vol. 35, no. 1-2, pp. 3-22, 1999.

[4] R. Agarwal, M. Bohner, D. O’Regan, and A. Peterson, "Dynamic equations on time scales: a survey," Journal of Computational and Applied Mathematics, vol. 141, no. 1-2, pp. 1-26, 2002.

[5] R. P. Agarwal, D. O’Regan, and S. H. Saker, "Philos-type oscillation criteria for second order half-linear dynamic equations on time scales," The Rocky Mountain Journal of Mathematics, vol. 37, no. 4, pp. 1085-1104, 2007.

[6] M. Bohner and A. Peterson, Dynamic Equations on Time Scales: An Introduction with Applications, Birkhäuser, Boston, Mass, USA, 2001.

[7] M. Bohner and A. Peterson, Eds., Advances in Dynamic Equations on Time Scales, Birkhäuser, Boston, Mass, USA, 2003.

[8] A. Del Medico and Q. Kong, "Kamenev-type and interval oscillation criteria for second-order linear differential equations on a measure chain," Journal of Mathematical Analysis and Applications, vol. 294, no. 2, pp. 621-643, 2004.

[9] A. Del Medico and Q. Kong, "New Kamenev-type oscillation criteria for second-order differential equations on a measure chain," Computers \& Mathematics with Applications, vol. 50, no. 8-9, pp. 1211-1230, 2005.

[10] O. Došlý and S. Hilger, "A necessary and sufficient condition for oscillation of the Sturm-Liouville dynamic equation on time scales," Journal of Computational and Applied Mathematics, vol. 141, no. 1-2, pp. 147-158, 2002.
[11] L. Erbe, A. Peterson, and S. H. Saker, "Oscillation criteria for second-order nonlinear dynamic equations on time scales," Journal of the London Mathematical Society. Second Series, vol. 67, no. 3, pp. 701-714, 2003.

[12] T. S. Hassan and Q. Kong, "Oscillation criteria for second order nonlinear dynamic equations with $p$-Laplacian and damping," Acta Mathematica Scientia B: English Edition, vol. 33, no. 4, pp. 975-988, 2013.

[13] H. Huang and Q.-R. Wang, "Oscillation of second-order nonlinear dynamic equations on time scales," Dynamic Systems and Applications, vol. 17, no. 3-4, pp. 551-570, 2008.

[14] Y.-C. Qiu and Q.-R. Wang, "Kamenev-type oscillation criteria of second-order nonlinear dynamic equations on time scales," Discrete Dynamics in Nature and Society, vol. 2013, Article ID 315158, 12 pages, 2013.

[15] Y.-C. Qiu and Q.-R. Wang, "Oscillation criteria of second-order dynamic equations with damping on time scales," Abstract and Applied Analysis, vol. 2014, Article ID 964239, 11 pages, 2014.

[16] S. H. Saker, R. P. Agarwal, and D. O'Regan, "Oscillation of second-order damped dynamic equations on time scales," Journal of Mathematical Analysis and Applications, vol. 330, no. 2, pp. 1317-1337, 2007.

[17] M. T. Şenel, "Kamenev-type oscillation criteria for the secondorder nonlinear dynamic equations with damping on time scales," Abstract and Applied Analysis, vol. 2012, Article ID 253107, 18 pages, 2012.

[18] Q.-R. Wang, "Oscillation criteria for nonlinear second order damped differential equations," Acta Mathematica Hungarica, vol. 102, no. 1-2, pp. 117-139, 2004.

[19] Q.-R. Wang, "Interval criteria for oscillation of certain second order nonlinear differential equations," Dynamics of Continuous, Discrete and Impulsive Systems Series A: Mathematical Analysis, vol. 12, no. 6, pp. 769-781, 2005. 


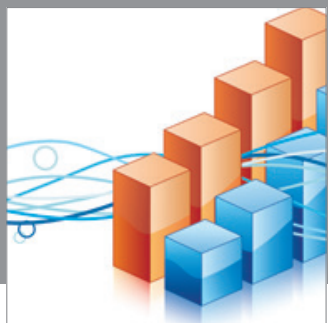

Advances in

Operations Research

mansans

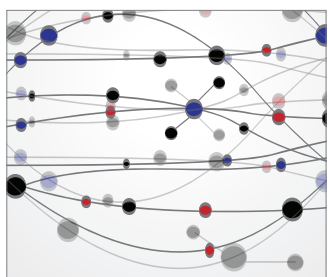

The Scientific World Journal
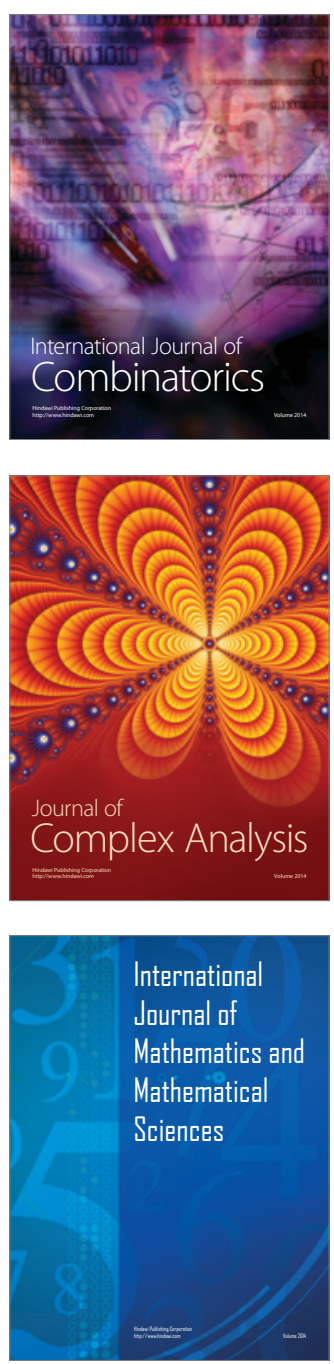
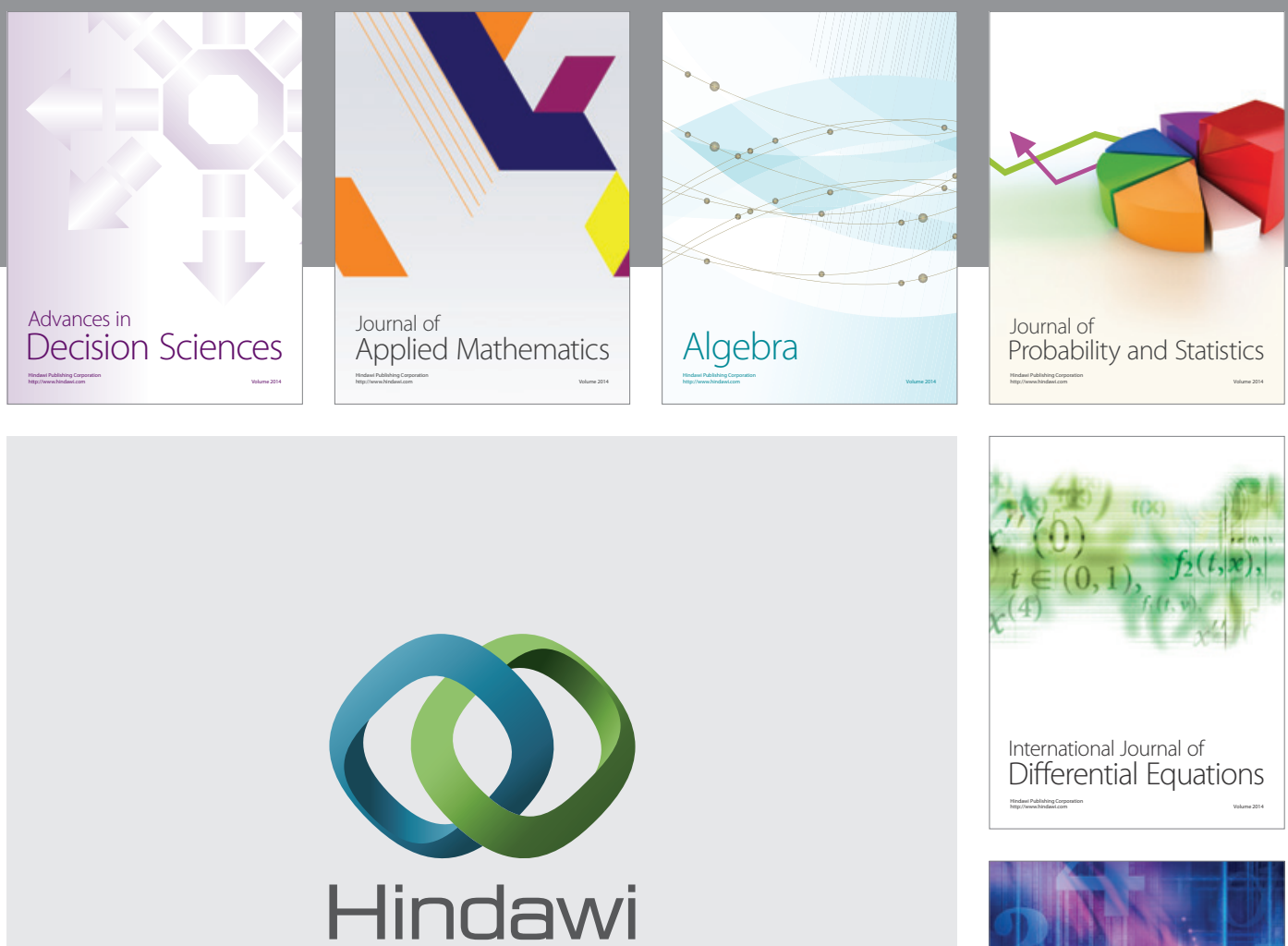

Submit your manuscripts at http://www.hindawi.com
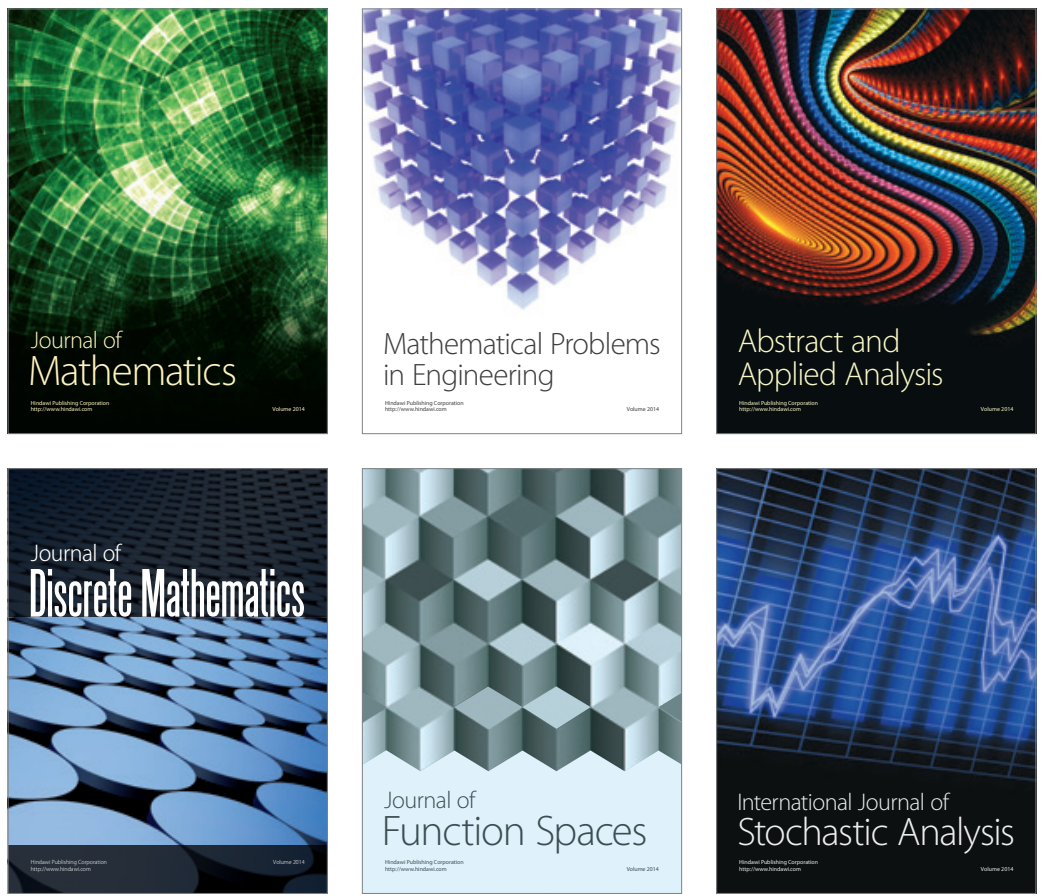

Journal of

Function Spaces

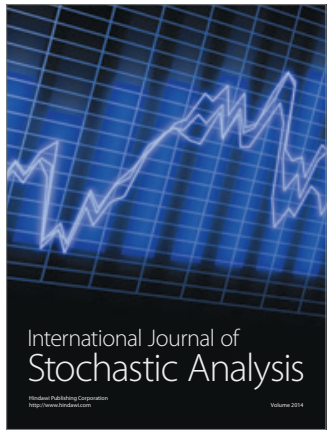

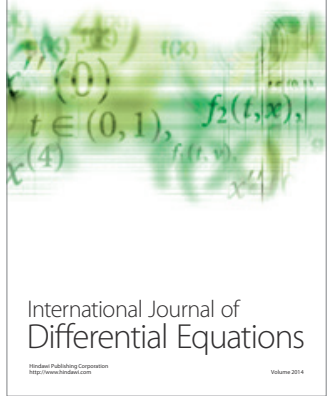
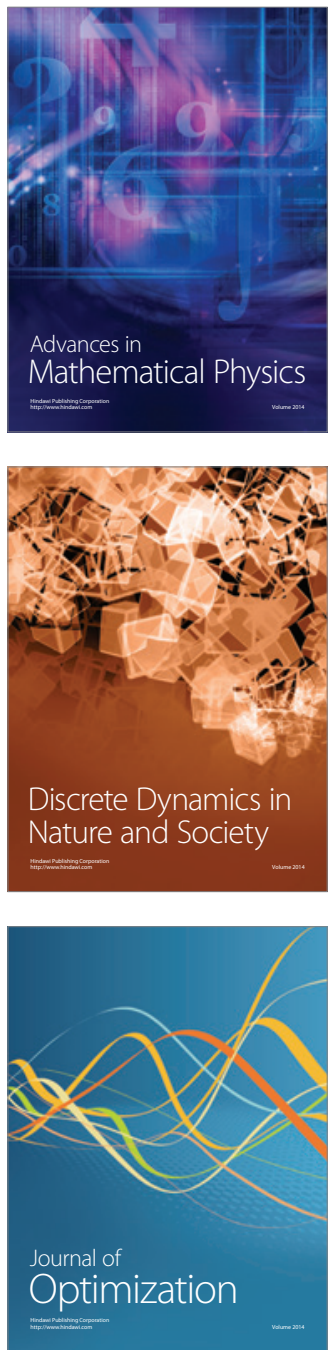